\title{
Effect of Wall Temperature on Roughness Induced Attachment-Line Transition
}

\author{
Anthony Dietz ${ }^{1}$, Colin Coleman ${ }^{2}, \operatorname{Jim~Laub}^{2}$, and D. I. A. Poll ${ }^{3}$ \\ 1 MCAT Inc., NASA Ames Research Center, Moffett Field, CA 94035, USA \\ 2 NASA Ames Research Center, Moffett Field, CA 94035, USA \\ 3 Cranfield College of Aeronautics, Cranfield University, Bedford MK43 OAL, UK
}

\begin{abstract}
An experiment on a cooled swept cylinder in a low-disturbance Mach 1.6 wind tunnel is described. The flow attachment line is disturbed by trip wires of varying size and the laminar/turbulent state of the downstream boundary layer is determined with a hot wire. The results demonstrate that although cooling the wall increases the stability of the boundary layer, it promotes roughness induced transition. Analysis of the data suggests that the attachment-line Reynolds number can account for the effect of wall cooling if the viscosity is evaluated at a particular reference temperature.
\end{abstract}

\section{Introduction}

Roughness induced transition is an important consideration in many design problems, from laminar flow wings to reentry thermal protection systems. The specification of allowable roughness levels not only influences the initial design, but has an impact on manufacturing and maintenance tolerances throughout the life of the vehicle. Hence, a reliable transition criteria that includes the effects of surface roughness is desired. Recent experiments have demonstrated that the boundary layer on a swept wing or body at incidence is most sensitive to roughness at the flow attachment line [1]. This is the dividing streamline that separates the flows over the upper and lower surfaces of the body. The attachment line exerts a strong influence on the downstream flow field as turbulence originating at the attachment line can corrupt downstream boundary layers. Therefore, the attachment line should be considered in the development of a transition criteria.

Poll [2] showed that transition data for incompressible attachment-line flows were correlated by the attachment-line Reynolds number:

$$
\bar{R}=V \eta / \nu
$$

where $V$ is the spanwise velocity (parallel to the attachment line) at the edge of the boundary layer, $\nu$ is the dynamic viscosity evaluated at the boundarylayer edge temperature and $\eta$ is a local length scale given by

$$
\eta=\sqrt{\frac{\nu}{(d u / d x)_{x=0}}}
$$


where $(d u / d x)_{x=0}$ is the chordwise velocity gradient (normal to the attachment line) evaluated at the edge of the boundary layer at the attachment line $(x=0)$. In a later paper, he suggested that the incompressible criteria could be extended to compressible flows by using a reference temperature similar to the one used successfully to extend incompressible skin friction and heat transfer correlations to compressible flows [3]. He proposed a reference temperature given by $T^{*}=T_{e}+A\left(T_{w}-T_{e}\right)+B\left(T_{r}-T_{e}\right)$ where $T_{e}$ is the boundary layer edge temperature, $T_{w}$ is the wall temperature and $T_{r}$ is the recovery temperature. $A$ and $B$ are empirically determined constants. Poll suggested values of $A=0.1$ and $B=0.6$ based on a correlation of the data available at that time. The data set has since been extended to higher Mach numbers. Some of the later tests, such as that by Arnal [1], produced results that supported the proposed criteria, while the results from others, eg Holden [4], have suggested a different reference temperature correlation should be chosen.

During transient flight conditions, such as those experienced during reentry, the wall temperature can be well below the recovery temperature. In these situations, a valid reference temperature is essential to the accurate application of the attachment-line transition criterion. In continuous flow tests where the attachment line is essentially adiabatic, the reference temperature reduces to $T^{*}=T_{e}+(A+B)\left(T_{r}-T_{e}\right)$. This demonstrates an inherent difficulty in testing the validity of the reference temperature correlation with near adiabatic models. Even in transient facilities, the tests are often limited to a single wall to total temperature ratio. When comparing tests in different facilities it is difficult to separate wall temperature effects from facility dependent effects such as freestream noise and model variations. In order to verify the effects of wall temperature variations on the reference temperature correlation, tests with a cooled model in a single facility are required. Such a test is reported here.

\section{Experimental Technique}

The aim of this experiment was to provide a data set with constant flow conditions but variable wall temperature. The experiment was performed on a 1.6-inch diameter cylinder mounted with a sweep angle of $76^{\circ}$ in the NASA Ames Mach 1.6 quiet wind tunnel. This facility was designed to provide continuous low-disturbance flow in a 8-inch $\mathrm{x} 16$-inch test section. The tunnel ran with air at ambient total temperature and pressures from 7 to $10 \mathrm{psia}$, giving a freestream Reynolds number range from $8.5 \mathrm{E} 6$ to $11.5 \mathrm{E} 6 \mathrm{Re} / \mathrm{m}$. A detailed description of the facility and its flow characteristics is given in [5]. Recent measurements of the test section flow quality were reported by Coleman et al. [6]. The rms pressure fluctuations at the center of the test section were less than $0.03 \%$ of the freestream total pressure for the frequency range $250 \mathrm{~Hz}$ to $50 \mathrm{kHz}$. The freestream turbulence intensity in this range was 
approximately $0.07 \%$. A schematic of the experimental arrangement is shown in Figure 1.

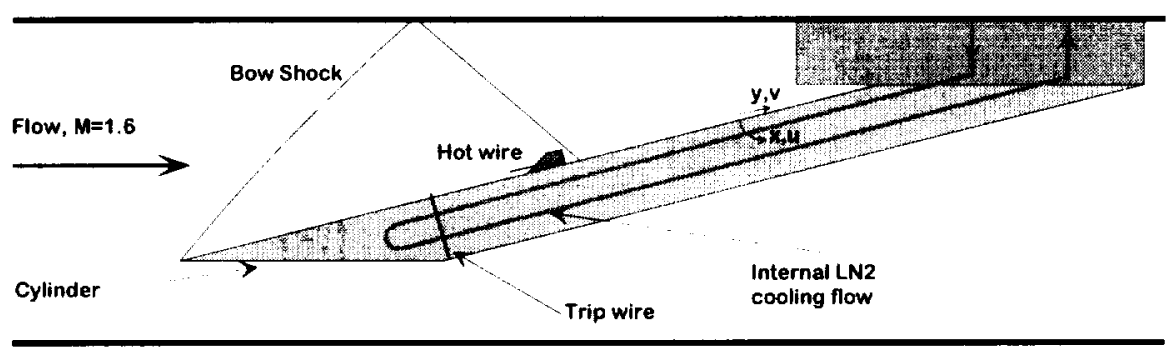

Fig. 1. Experimental set up

Two swept cylinder models of identical dimensions were used in this investigation. The first model was fitted with pressure taps along the attachment line and also along lines normal to the attachment line at five spanwise stations. This model was used to measure the pressure gradient along the attachment line to determine the extent of leading-edge effects and the location of the reflected bow shock. It was also used to measure the chordwise pressure distribution. The second model was used for the transition tests. It consisted of a highly polished outer shell with a $1 / 8$ " thick wall, and an inner cooling plug with passages through which liquid nitrogen could be pumped to cool the cylinder. The outer shell was instrumented with 10 thermocouples, mounted from the inside, 0.02 " below the external surface and spaced evenly along the attachment line. The boundary layer was tripped with stainlesssteel music wire. The wire was wrapped around the cylinder, tensioned until it was close to its yield stress, and fixed in place with low temperature epoxy applied to the rear side of the cylinder. A $2.5 \mu \mathrm{m}$ surface-mounted hot wire was used to measure the state of the attachment-line boundary layer downstream of the trip. Surface icing was a concern because the test section air was dried but not heated and so for reasonable temperature ratios, the cylinder wall temperature was well below the dew point. In order to minimize icing effects, the cylinder surface was cleaned and polished before each run and the trip itself was also cleaned before being fixed in place. During the tests, the amount of ice and time of formation was closely monitored and recorded.

\section{Experimental Conditions}

The experimental configurations for the data presented here are given in Table 1. Measurements were made with the cylinder mounted at two locations in the test section, and repeat tests were made with the trip and hot wire mounted at varying $y / D$ locations to test for the effect of variations in the 
spanwise pressure gradient along the cylinder. A plot of the pressure coefficients measured along the attachment line is shown in Figure 2. The first series of tests were made with the cylinder in the forward, high position. In this configuration, the measurement range was limited by the leading edge pressure gradient at the front $(y / D<4.5)$, and by the pressure rise due to the reflected bow shock at the rear $(y / D<5.7)$. To determine whether the spanwise pressure variations were significant, some measurements were repeated with the cylinder in a rear low position where the reflected bow shock was further back giving a longer measurement region.

Table 1. Test Matrix

\begin{tabular}{lllll}
\hline Run No. & Model Position & Trip y/D & Hot Wire y/D & Trip Size (in) \\
\hline a1r07c01 & fwd high & 5.00 & 5.31 & 0.009 \\
a1r12c01 & fwd high & 4.69 & 5.31 & 0.010 \\
a1r13c01 & fwd high & 4.69 & 5.00 & 0.010 \\
a1r15c01 & fwd high & 4.69 & 5.31 & 0.008 \\
a1r16c01 & fwd high & 4.69 & 5.31 & 0.007 \\
a3r02c01 & rear low & 5.63 & 6.56 & 0.009 \\
a3r03c01 & rear low & 4.69 & 6.56 & 0.009 \\
\hline
\end{tabular}

The attachment-line Reynolds number was calculated using boundarylayer edge conditions determined from the measured freestream conditions by assuming the angle of the bow shock required to give the measured surface pressure coefficient. The chordwise velocity gradient was calculated from the measured chordwise pressure distribution. The total temperature was typically $295 \mathrm{~K}$, resulting in a boundary-layer edge temperature of $180 \mathrm{~K}$. The wall cooling could be varied from adiabatic to $150 \mathrm{~K}$, giving wall to total temperature ratios from 0.95 to 0.5 .

\section{Results}

A similar technique was used in each test. First, the tunnel was run for about 20 minutes until the model and tunnel walls had cooled to an equilibrium temperature. The total pressure was then increased until transition was observed on the near adiabatic model. A number of lower pressures were then set, and at each pressure, the model was cooled until transition was observed. The cooling was then halted and the model was allowed to warm until the layer relaminarized. At least two cooling attempts were made at each pressure. The results of a typical test run are shown in Figure 3. 


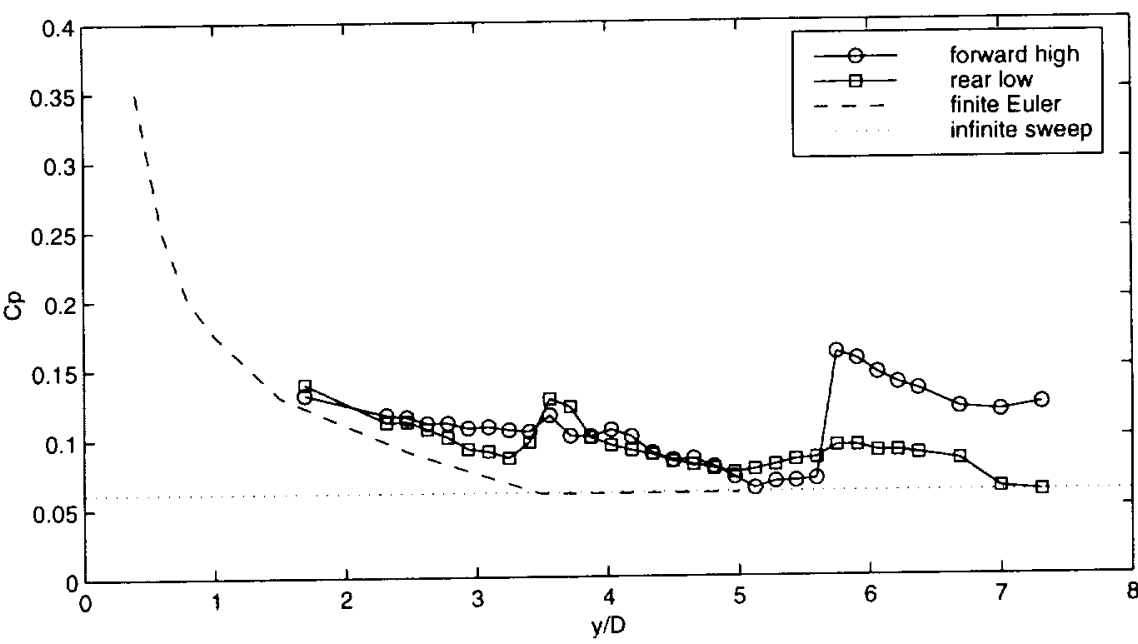

Fig. 2. Surface pressure coefficient measured along the attachment line with the cylinder in the forward/low and rear/high locations in the test section. Results of an Euler calculation for the finite length cylinder are also shown along with the infinite swept value.

The effect of cooling on transition can be seen in the figure. For a certain trip height, wall cooling causes transition at a lower freestream Reynolds number. The transition points were repeatable as long as the trip was applied under sufficient tension. Loose trips resulted in significant hysteresis in the transition temperature between cooling and warming. The figure also shows that the thin layer of ice seen on the model at low temperatures did not affect the transition results. Repeatable results were also obtained when the cylinder was moved from the forward to the rear location, indicating that slight variations in pressure coefficient along the cylinder did not significantly affect the results. Data from all the runs are presented in Figure 4a in terms of the at tachment line Reynolds number and the non-dimensional roughness height. Contours of the wall to total temperature ratio are also included in the plot. The sensitivity of the roughness induced transition mechanism to wall temperature is evident. This sensitivity remained in the $\overline{R^{*}}$ Reynolds numbers calculated using Poll's reference temperature. However, if the wall temperature coefficient, $A$, in the reference temperature formulation is increased from 0.1 to 0.62 , the sensitivity is removed. This result is shown in Figure $4 \mathrm{~b}$. The optimum value of $A$ depends on the value of the compressibility coefficient, $B$. As $B$ is increased from 0 to 1 , the optimum value of $A$ increases linearly from 0.62 to 0.92 . As the measurements reported here were all made at a constant Mach number, no conclusions can be made about the correct value of the coefficient $B$. The data in Figure $4 \mathrm{~b}$ are plotted with $B=0$. 

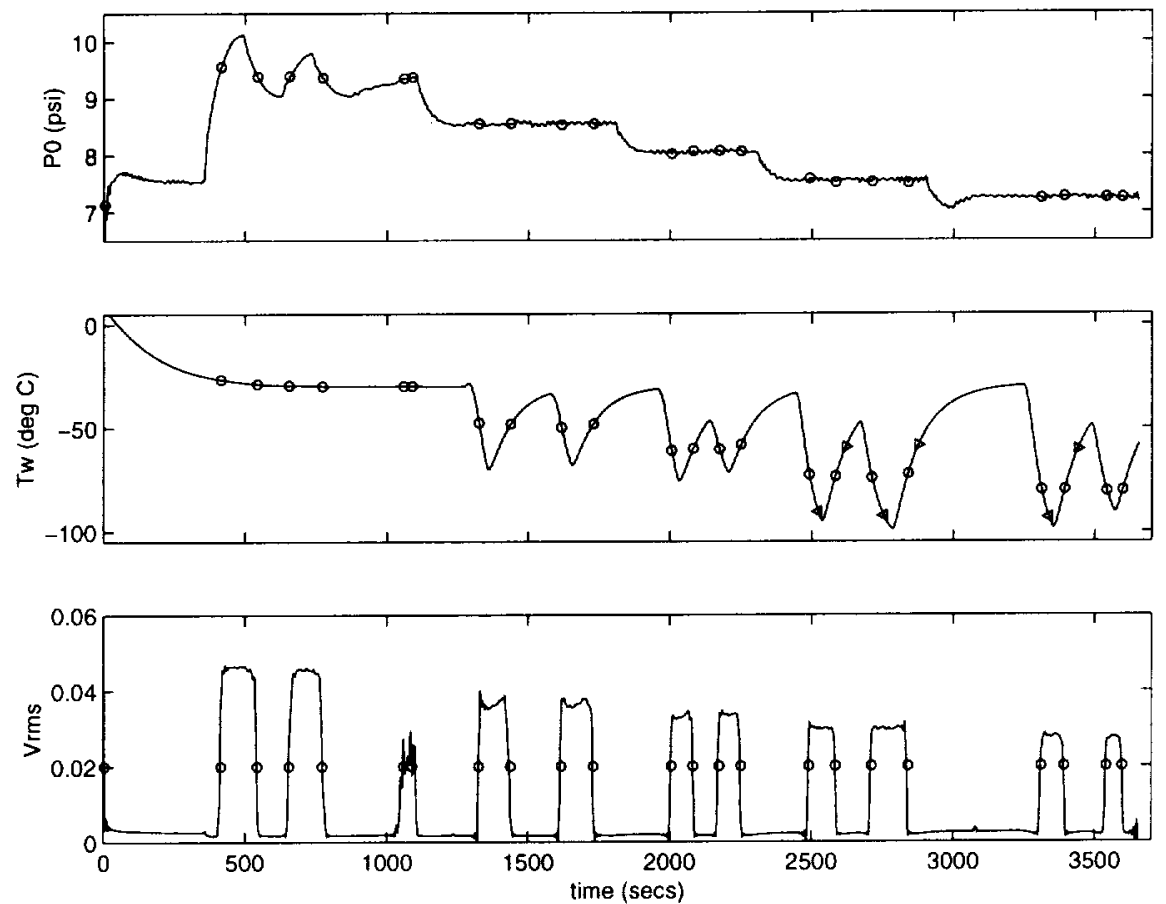

Fig. 3. Traces of the total pressure, wall temperature and hot wire rms Voltage from run a1r07c01. Transition points are depicted by circles and the occurrence of ice by the arrows.
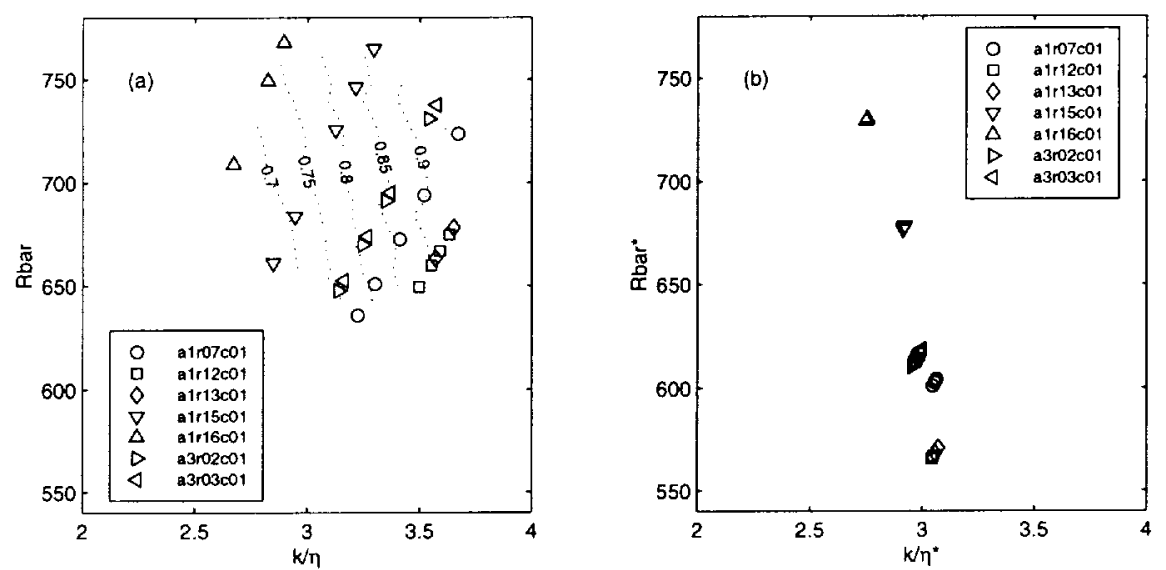

Fig. 4. Effect of wall temperature on attachment line Reynolds number. (a) Reynolds number and $\eta$ evaluated using boundary layer edge temperature. Contours of $T_{w} / T_{0}$ also shown (b) $\overline{R^{*}}$ and $\eta^{*}$ evaluated using reference temperature $T^{*}=T_{e}+0.62\left(T_{w^{\prime}}-T_{e}\right)$ 


\section{Discussion}

There are two competing mechanisms at work in a cooled attachment line boundary layer. Cooling increases the stability of the attachment line and delays smooth wall transition [6]. However, cooling reduces the boundary-layer thickness, increasing the relative height of the roughness. These two effects are illustrated in Figure 5, where plots of the power spectral density of the hot wire signal are shown. The plots were recorded downstream of the same trip at three wall temperatures. Increased energy in the unstable frequency range is apparent in the data recorded at $-21^{\circ}$. When the wall is cooled to $-55^{\circ}$ the instability waves are suppressed. However, as the cooling is increased further, the layer suddenly goes turbulent and a flat spectrum results at $-65^{\circ}$. So although cooling increases the stability of the boundary layer, it promotes the bypass transition mechanism associated with roughness. As the temperature was reduced further, the boundary layer remained turbulent and no effect of cooling on the ability of the attachment layer to sustain turbulence was observed. The Reynolds number range in this experiment may have been too high to observe such an effect. Roquefort [7] did observe a delay in transition associated with gross contamination in his experiments with a cooled swept cylinder at lower Reynolds numbers.

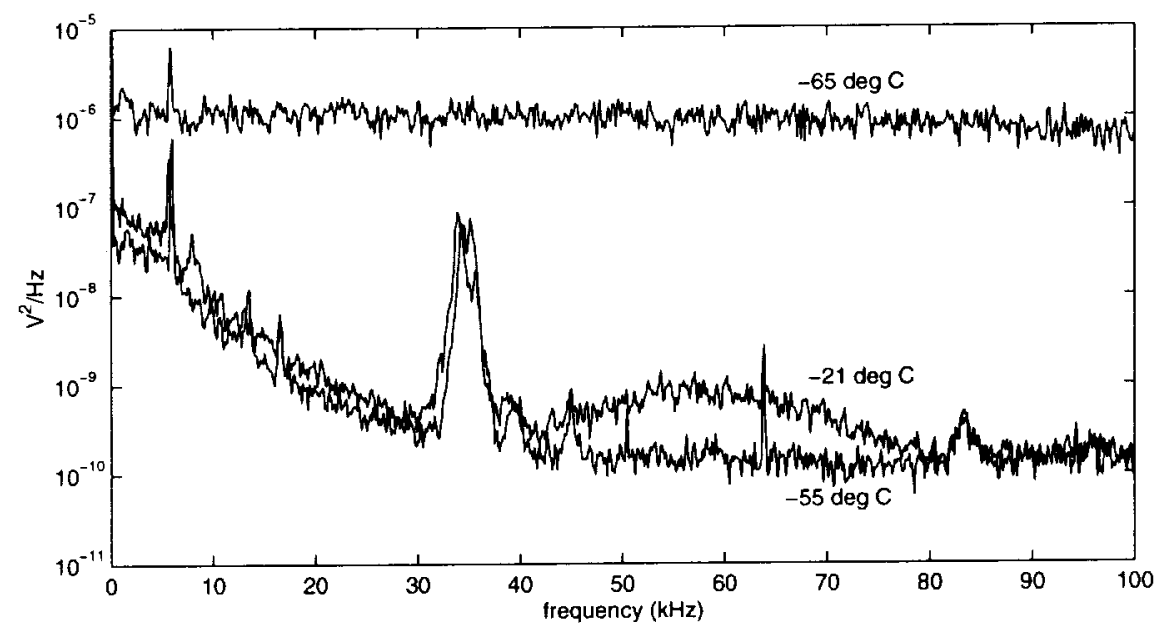

Fig. 5. Power spectral density of the hot-wire signal recorded at three different wall temperatures during run $\mathrm{A} 3 \mathrm{r} 03 \mathrm{c} 01$.

The transition Reynolds numbers measured during this test, and previously by Coleman [6] are higher than those predicted by Poll's criteria and reported from other investigations. Although an attempt was made to move further from the leading edge of the cylinder, Figure 1 suggests there may still 
be some leading edge influence. However, Reynolds numbers computed using local conditions should still be valid. The other difference between this and previous experiments is the low-disturbance nature of the freestream flow. This may have contributed to the high transition Reynolds numbers.

\section{Conclusions}

Results from an experiment on a swept cylinder at Mach 1.6 have demonstrated the effects of wall temperature on roughness-induced attachment-line transition. Although wall cooling damps viscous instabilities in the boundary layer, it promotes the bypass transition mechanism associated with roughness. Analysis of the data suggests that the transition Reynolds number, $\overline{R^{*}}$, can account for the effects of wall cooling if the viscosity is evaluated at the correct reference temperature. For this experiment, the optimum wall temperature coefficient, $A$, in the reference temperature formulation, $T^{*}=T_{e}+A\left(T_{w}-T_{e}\right)+B\left(T_{r}-T_{e}\right)$, varies from 0.62 and 0.92 for values of the compressibility coefficient $B$ between 0 and 1 .

\section{References}

1. Arnal, D., Vignau, F., and Juillen, J. C. (1989) Boundary layer tripping in supersonic flow. Proceedings of the IUATM Symposium on Laminar-Turbulent Transition. Springer-Verlag, Berlin, 1-23

2. Poll D. I. A. (1979) Transition in the infinite swept attachment line boundary layer. Aero Q. 30, 607-629

3. Poll D. I. A. (1983) The development of intermittent turbulence on a swept attachment line including the effects of compressibility. Aero Q. 34, 1-23

4. Holden, M. and Kolly, J. (1995) Attachment line transition studies on swept cylindrical leading edges at Mach numbers from 10 to 12. AIAA Paper 95-2279

5. Wolf, W. D. and Laub, J. A. (1996) Low disturbance flow characteristics of the NASA-Ames Laminar Flow Supersonic Wind Tunnel. AIAA Paper 96-2189

6. Coleman, C. P., Poll, D. I. A., and Lin, R. -S. (1997) Experimental and computational investigation of leading edge transition at Mach 1.6. AIAA Paper 97-1776

7. Alziary de Roquefort, T. (1989) Leading Edge Transition in Hypersonic Flows. Hypersonics, Volume II, Computation and Measurement of Hypersonic Flows, Birkhauser, Boston, 151-180 